\title{
Cobalt(II) Chloride Catalyzed one Pot Synthesis of 2-substituted and 3-substituted-4(3H)-Quinazolinones
}

\author{
AAYESHA NASREEN* and RITA M. BORIK \\ Department of Chemistry, College of Sciences, Jazan University, \\ 6811-Arroudah P.O Box No. 2097, Jazan 82724-3750, Saudi Arabia. \\ ${ }^{*}$ Corresponding author E-mail: aayesha_iict@yahoo.co.in \\ http://dx.doi.org/10.13005/ojc/300249
}

(Received: February 15, 2014; Accepted: March 25, 2014)

\begin{abstract}
Cobalt(II) chloride (10mol\%) was found to be an efficient catalyst for one pot synthesis of variety of 2-substituted-4 $(3 \mathrm{H})$ quinazolinones by condensation of anthranilamide and aldehydes and synthesis of 3-substituted-4(3H)quinazolinones by condensation of anthranilic acid, orthoester, primary amines at reflux giving good to excellent yields (75-95\%).
\end{abstract}

Key words: Cobalt(II) chloride, 2-substituted and 3-substituted 4(3H)quinazolinones, Anthranilamide, aldehydes, anthranilic acid, $\mathrm{HC}(\mathrm{OEt})_{3}$, anilines.

\section{INTRODUCTION}

The quinazolinone core and its derivatives form an important class of compounds, as they are present in a large family of products with broad biological activities. 4(3H)-quinazolinones are versatile nitrogen heterocyclic compounds, displaying a broad spectrum of biological and pharmacological activities such as anti-fungal, ${ }^{1}$ antitumour, ${ }^{2}$ hypotensive, ${ }^{3}$ anti-cancer, ${ }^{4,5}$ anti-HIV, ${ }^{6}$ antiinflammatory, ${ }^{7}$ anti-bacterial, ${ }^{8}$ etc. Furthermore, $4(3 \mathrm{H})$-quinazolinones substituted at 2,3-position derivatives play a pivotal role in the hypertensive activity. ${ }^{9,10}$
Several bioactive natural products such as febrifugine and isofebrifugine contain quinazolinone moieties with potential anti-malarial activity ${ }^{11}$ Similarly quinazolinone containing moieties have been known as tyrosine kinase inhibitors, ${ }^{12}$ dihydrofolate reductase inhibitors, ${ }^{13}$ and tubulin polymerization inhibitors. ${ }^{14}$ Due to their wide range of applications these compounds have received a great deal of attention in connection with their synthesis.

Many reagents have been reported in the literature, ${ }^{15-26}$ for the synthesis of $4(3 \mathrm{H})$ quinazolinone derivatives. Among these methods 2-substituted-4(3H)quinazolinones have been 
synthesized from anthranilamide and aldehydes using $\mathrm{NaHSO}_{3}{ }^{16 \mathrm{a}} \mathrm{DDQ},{ }^{16 \mathrm{~b}} \mathrm{CuCl}_{2},{ }^{20} \mathrm{FeCl}_{3} \cdot 6 \mathrm{H}_{2} \mathrm{O},{ }^{25 \mathrm{a}}$ $\mathrm{Cu}\left(\mathrm{NO}_{3}\right)_{2} \cdot 3 \mathrm{H}_{2} \mathrm{O},{ }^{25 \mathrm{~b}}$ and 3 -substituted-4(3H) quinazolinones were recently prepared by using various catalysts such as $\mathrm{Yb}(\mathrm{OTf})_{3}{ }^{15 \mathrm{~h}}$ Silicagel/ $\mathrm{FeCl}_{3,}{ }^{17 \mathrm{a}}$ Nafion- $\mathrm{H},{ }^{17 b}$ (a perfluorinated resin supported sulfonic acid), under microwave irradiation, $\mathrm{Zn}\left(\mathrm{ClO}_{4}\right)_{2}{ }^{18}$ heteropolyacids such as $\mathrm{H}_{3} \mathrm{PW}_{12} \mathrm{O}_{40} \cdot 13 \mathrm{H}_{2} \mathrm{O},{ }^{19} \mathrm{La}\left(\mathrm{NO}_{3}\right)_{3} \cdot 6 \mathrm{H}_{2} \mathrm{O},{ }^{21} \mathrm{Bi}(\mathrm{TFA})_{3}{ }^{-}$ $\mathrm{FeCl}_{4}{ }^{22}$

In continuation of our work to develop new organic transformations, ${ }^{27-29}$ we report here in that cobalt(II) chloride which acts as a mild lewis acid might be useful and inexpensive catalyst for the synthesis of 2-substituted-4(3H)quinazolinones and 3-substituted-4(3H)quinazolinones. Although cobalt(II) chloride has been extensively used as a mild catalyst for a variety of organic transformations, ${ }^{30-32}$ there are no examples of the use of cobalt chloride as catalyst for the synthesis of 2-substituted-4(3H)-quinazolinones and 3substituted-4(3H)quinazolinones.

In the present study 2-substituted$4(3 \mathrm{H})$ quinazolinones and 3 -substituted-4(3H) quinazolinones are synthesized and the results are presented here. A high yield condensation of anthranilamide, aldehyde (alkyl, aryl, heteroaryl) acetonitrile at reflux in the presence of catalytic amount of $\mathrm{co}(\mathrm{II})$ chloride 2-substituted$4(3 \mathrm{H})$ quinazolinones are obtained(scheme-1). 3substituted-4(3H)quinazolinones are obtained from the reaction of anthranilic acid, triethyl ortho-formate $\left(\mathrm{HC}(\mathrm{OEt})_{3}\right)$ and anilines at reflux in the presence of catalytic amount of $\mathrm{co}$ (II) chloride and acetonitrile as solvent(scheme-2).

\section{MATERIAL AND METHODS}

Chemicals were purchased from Merck and Fluka and directly used for the synthesis. Thin layer chromatography (TLC): precoated silica gel plates (60 F254, 0.2mm layer; E. Merck). ${ }^{1} \mathrm{H}$ NMR (Avance $300 \mathrm{MHz}$ ) spectra were recorded in DMSO using TMS as internal standard. Chemical shifts (') are reported in ppm, Melting points (M.P.) were determined on a Fischer-Johns melting point apparatus. IR and MS were recorded on a Thermo Nicolet Nexus 670 FT-IR Spectrometer and
Finnegan MAT 1020 Mass spectrometer operating at $70 \mathrm{ev}$. Elemental analyses were performed on a Perkin Elmer 2400series II Elemental CHN analyzer.

General procedure for the synthesis of 2substituted-4(3H)quinazolinones

To a mixture of anthranilamide (1 $\mathrm{mmol})$ in acetonitrile $(5 \mathrm{~mL}), \mathrm{CoCl}_{2}(10 \mathrm{~mol} \%)$ was added to the appropriate aldehyde $(1.3 \mathrm{mmol})$ was refluxed at $70^{\circ} \mathrm{C}$ for the time specified in (Table-3) for each substrate, after completion of the reaction as indicated by TLC the reaction mixture was allowed to cool and quenched with $\mathrm{NaHCO}_{3}$ followed by brine solution and extracted with ethyl acetate dried over $\mathrm{Na}_{2} \mathrm{SO}_{4}$, concentrated under vacuum, and the crude mixture was purified by column chromatography (hexane:ethylacetate $7: 3$ ) to afford the corresponding pure 2-substituted$4(3 \mathrm{H})$ quinazolinones $3 \mathrm{a}-3 \mathrm{~m}$ (Table-3). All the products are well characterized by spectral analysis (IR, ${ }^{1} \mathrm{HNMR}, \mathrm{MS}$ ) and were found to be identical those reported in the literature. ${ }^{20,25}$

General procedure for the synthesis of 3substituted-4(3H)quinazolinones

To a mixture of anthranilic acid $(1 \mathrm{mmol})$ in acetonitrile $(5 \mathrm{~mL})$ triethyl orthoformate $\left(\mathrm{HC}(\mathrm{OEt})_{3}\right)$ $1.5 \mathrm{mmol})$, the appropriate aniline $(1.3 \mathrm{mmol})$ and $\mathrm{CoCl}_{2}(10 \mathrm{~mol} \%)$ was refluxed at $70{ }^{\circ} \mathrm{C}$ for the time specified in (Table-4) for each substrate, after completion of the reaction as indicated by TLC the reaction mixture was allowed to cool and quenched with $\mathrm{NaHCO}_{3}$ followed by brine solution and extracted with ethyl acetate dried over $\mathrm{Na}_{2} \mathrm{SO}_{4}$, concentrated under vacuum, and the crude mixture was purified by column chromatography (hexane:ethylacetate $7: 3$ ) to afford the corresponding pure 3-subtituted-4(3H) quinazolinones 7a-7o (Table-4). All the products are well characterized by spectral analysis (IR, ${ }^{1} \mathrm{HNMR}, \mathrm{MS}$ ) and were found to be identical with those reported in the literature. ${ }^{14 b, 17-19,21,26}$

Spectral data for selected compounds: 2-Phenyl4(3H)-quinazolinone (3a)

$\mathrm{mp}\left({ }^{\circ} \mathrm{C}\right)^{25}$ 237-239, IR(KBr): 679, 1458, 180, 1356, 1037, 965,738 $\mathrm{cm}^{-1} ;{ }^{1} \mathrm{HNMR}(300 \mathrm{MHz}$, DMSO$\left.\mathrm{d}_{6}\right)$ 7.56-7.62 (m, 4H),7.75-7.84 (m, 2H), 8.18-8.53 $(\mathrm{m}, 3 \mathrm{H}), 12.46(\mathrm{~s}, 1 \mathrm{H}, \mathrm{NH}) \mathrm{ppm}$; Anal. Calcd. for $\mathrm{C}_{14} \mathrm{H}_{10} \mathrm{~N}_{2} \mathrm{O}: \mathrm{C}, 75.68 ; \mathrm{H}, 4.51 ; \mathrm{N}, 12.68 ; \mathrm{O}, 7.5$. Found: 
C, 75.67; H, 4.48; N, 12.50. MS(EI): m/z (\%) 222(M+).

\section{2-(4-Hydroxyphenyl-3-methoxy)-4(3H)-} quinazolinone (3c)

$\mathrm{mp}\left({ }^{\circ} \mathrm{C}\right)^{25} 267-268, \mathrm{IR}(\mathrm{KBr}) 1664,1578$, $1545,1483,1280,1248,1027,1025,866,770 \mathrm{~cm}-$ 1; ${ }^{1} \mathrm{HNMR}\left(300 \mathrm{MHz}, \mathrm{DMSO}-\mathrm{d}_{6}\right) 3.94\left(\mathrm{~s}, 3 \mathrm{H}, \mathrm{CH}_{3}\right)$, $6.98(\mathrm{~d}, 1 \mathrm{H}, \mathrm{J}=8: 3 \mathrm{~Hz}, \mathrm{Ph}), 7.44(\mathrm{t}, 1 \mathrm{H}, \mathrm{J}=7: 4 \mathrm{~Hz}, \mathrm{Ph})$, 7.75 (d, 1H, J=8:0Hz, Ph), 7.77 (d, 1H, J=8:3Hz,Ph), $7.80(\mathrm{~s}, 1 \mathrm{H}, \mathrm{Ph}), 7.86(\mathrm{t}, 1 \mathrm{H}, \mathrm{J}=7: 2 \mathrm{~Hz}, \mathrm{Ph}), 8.17$ (d, $1 \mathrm{H}, \mathrm{J}=7: 8 \mathrm{~Hz}, \mathrm{Ph}$ ), 9.85 (s, $1 \mathrm{H}, \mathrm{OH}) ; 12.38(\mathrm{~s}, 1 \mathrm{H}$, $\mathrm{NH}$ ) ppm; Anal. Calcd for $\mathrm{C}_{15} \mathrm{H}_{12} \mathrm{~N}_{2} \mathrm{O}_{3}$ : C, 67.20; $\mathrm{H}$, 4.53; N, 10.49. Found: C, 67.29; H, 4.60; N,10.30; MS (EI): $m / z(\%)$ 268. $\left(M^{+}\right)$

\section{2-(4-Nitrophenyl)-4(3H)-quinazolinone (3i)} $\mathrm{mp}\left({ }^{\circ} \mathrm{C}\right)^{25}>300, \mathrm{IR}(\mathrm{KBr}): 1682,1609,1590$, 1523, 1469, 1348, 1151, $949,865,772,707,556$ $\mathrm{cm}^{-1}$; ${ }^{1} \mathrm{HNMR}\left(300 \mathrm{MHz}\right.$, DMSO-d $\left.\mathrm{d}_{6}\right) 7.59(\mathrm{t}, 1 \mathrm{H}$, $\mathrm{J}=7: 5 \mathrm{~Hz}, \mathrm{Ph}), 7.80(\mathrm{~d}, 1 \mathrm{H}, \mathrm{J}=8: 0 \mathrm{~Hz}, \mathrm{Ph}), 7.89(\mathrm{t}$, $1 \mathrm{H}, \mathrm{J}=7: 6 \mathrm{~Hz}, \mathrm{Ph}), 8.19$ (d, $1 \mathrm{H}, \mathrm{J}=8: 1 \mathrm{~Hz}, \mathrm{Ph}), 8.38$ (d, $2 \mathrm{H}, \mathrm{J}=9: 1 \mathrm{~Hz}, \mathrm{Ph}), 8.43(\mathrm{~d}, 2 \mathrm{H}, \mathrm{J}=9: 1 \mathrm{~Hz}, \mathrm{Ph})$ 12.16(s, $1 \mathrm{H}, \mathrm{NH})$ ppm; Anal. Calcd for $\mathrm{C}_{14} \mathrm{H}_{9} \mathrm{~N}_{3} \mathrm{O}_{3}$ : C, 62.94; H, 3.40; N, 15.72, Found: C, 62.79; H,3.56; $\mathrm{N}, 15.89 ; \mathrm{MS}(\mathrm{El}): \mathrm{m} / \mathrm{z}(\%) 267\left(\mathrm{M}^{+}\right)$.

\section{2-(Furyl)-4(3H)-quinazolinone (3f)}

$\mathrm{mp}\left({ }^{\circ} \mathrm{C}\right)^{25} 219-221, \mathrm{IR}(\mathrm{KBr}): 1663,1618$, $1569, \quad 1457,1330,969,775,757 \mathrm{~cm}^{-1}$; ${ }^{1} \mathrm{HNMR}(300 \mathrm{MHz}$, DMSO-d $)$ 6.73(dd, $1 \mathrm{H}, \mathrm{J}=3: 4$, $1.3 \mathrm{~Hz}), 7.49(\mathrm{t}, 1 \mathrm{H}, \mathrm{J}=7: 5 \mathrm{~Hz}, \mathrm{Ph}), 7.59(\mathrm{~d}, 1 \mathrm{H}$, $\mathrm{J}=3: 4 \mathrm{~Hz}), 7.68(\mathrm{~d}, 1 \mathrm{H}, \mathrm{J}=8: 1 \mathrm{~Hz}, \mathrm{Ph}), 7.81(\mathrm{t}, 1 \mathrm{H}$, $\mathrm{J}=7: 6 \mathrm{~Hz}, \mathrm{Ph}), 7.97(\mathrm{~d}, 1 \mathrm{H}, \mathrm{J}=1: 3 \mathrm{~Hz}), 8.11(\mathrm{~d}, 1 \mathrm{H}$, $\mathrm{J}=7: 8 \mathrm{~Hz}, \mathrm{Ph}), 12.68(\mathrm{~s}, 1 \mathrm{H}, \mathrm{NH}) \mathrm{ppm}$; Anal. Calcd for $\mathrm{C}_{12} \mathrm{H}_{8} \mathrm{~N}_{2} \mathrm{O}_{2}: \mathrm{C}, 67.98 ; \mathrm{H}, 3.76 ; \mathrm{N}, 13.22$. Found: $\mathrm{C}$, 67.86; H, 3.79; N, 13.26; MS(EI): m/z (\%) 212(M+).

\section{3-Phenyl-4(3H)-quinazolinone (7a)}

$\mathrm{mp}\left({ }^{\circ} \mathrm{C}\right)^{19} 139, \mathrm{IR}(\mathrm{KBr}): 1699,1598,1037$, 1290, 1463, $\mathrm{cm}^{-1} ;{ }^{1} \mathrm{HNMR}(300 \mathrm{MHz}$, DMSO-d $)$ ): 8.34 $(\mathrm{d}, \mathrm{J}=7.6 \mathrm{~Hz}, 1 \mathrm{H}), 8.16(\mathrm{~s}, 1 \mathrm{H}), 7.72-7.78(\mathrm{~m}, 2 \mathrm{H})$, $7.51(\mathrm{t}, \mathrm{J}=7.31 \mathrm{~Hz}, 1 \mathrm{H})$ and 7.28-7.36 (m, 5H) ppm; Anal. Calcd. for $\mathrm{C}_{14} \mathrm{H}_{10} \mathrm{~N}_{2} \mathrm{O}: \mathrm{C}, 75.69 ; \mathrm{H}, 4.52 ; \mathrm{N}, 12.65$; $\mathrm{O}, 7.2$. Found: $\mathrm{C}, 75.60 ; \mathrm{H}, 4.44 ; \mathrm{N}, 12.59 ; \mathrm{MS}(\mathrm{EI}): \mathrm{m} /$ z (\%) $222\left(\mathrm{M}^{+}\right)$.

\section{3-(4-Bromophenyl)-4(3H)-quinazolinone (7c) $\mathrm{mp}\left({ }^{\circ} \mathrm{C}\right)^{19} 186, \mathrm{IR}(\mathrm{KBr}): 1692,1605,1456$, $1070 \mathrm{~cm}^{-1} ;{ }^{1} \mathrm{HNMR}\left(300 \mathrm{MHz}\right.$, DMSO-d $\left.\mathrm{d}_{6}\right): 8.43(\mathrm{~d}, \mathrm{~J}=$ $8.6 \mathrm{~Hz}, 1 \mathrm{H}), 8.12(\mathrm{~s}, 1 \mathrm{H})$ and $7.95-7.21(\mathrm{~m}, 7 \mathrm{H})$}

ppm; Anal. Calcd. for $\mathrm{C}_{14} \mathrm{H}_{9} \mathrm{BrN}_{2} \mathrm{O}: \mathrm{C}, 55.83 ; \mathrm{H}, 3.09$; $\mathrm{Br}, 26.55 ; \mathrm{N}, 9.30$. Found: C, 55.92; H, 3.07; N, 9.31; $M S(E I): m / z(\%), 300\left(M^{+}\right)$.

\section{3-(4-Methylphenyl)-4(3H)-quinazolinone (7f)} $\mathrm{mp}\left({ }^{\circ} \mathrm{C}\right)^{19} 148, \mathrm{IR}(\mathrm{KBr}): 1690,1578,1603$, $1457 \mathrm{~cm}^{-1} ;{ }^{1} \mathrm{HNMR}\left(300 \mathrm{MHz}, \mathrm{DMSO}-\mathrm{d}_{6}\right): 8.29(\mathrm{~d}, \mathrm{~J}=$ $7.6 \mathrm{~Hz}, 1 \mathrm{H}), 8.11(\mathrm{~s}, 1 \mathrm{H}), 7.69-7.71(\mathrm{~m}, 2 \mathrm{H}), 7.40(\mathrm{t}$, $\mathrm{J}=7.2 \mathrm{~Hz}, 2 \mathrm{H}), 7.16(\mathrm{~d}, \mathrm{~J}=7.6 \mathrm{~Hz}, 2 \mathrm{H}), 7.28(\mathrm{~d}, \mathrm{~J}=$ $7.6 \mathrm{~Hz}, 2 \mathrm{H}$ ) and 2.26 (s, 3H)ppm; Anal. Calcd. for $\mathrm{C}_{15} \mathrm{H}_{12} \mathrm{~N}_{2} \mathrm{O}: \mathrm{C}, 76.25 ; \mathrm{H}, 5.17 ; \mathrm{N}, 11.88 ; \mathrm{O}, 6.97$. Found: C, 76.29; H, 5.16; N, 11.77; MS(EI): $\mathrm{m} / \mathrm{z}$ (\%) $236\left(\mathrm{M}^{+}\right)$.

\section{3-(2,5-Dichlorophenyl)-4(3H)-quinazolinone (7h) $\mathrm{mp}\left({ }^{\circ} \mathrm{C}\right)^{19} \quad 146-148 \quad \mathrm{IR}(\mathrm{KBr}): 1673$,} $1604,15781450 \mathrm{~cm}^{-1} ;{ }^{1} \mathrm{HNMR}(300 \mathrm{MHz}$, DMSO-d 6 ): $8.43(\mathrm{~d}, \mathrm{~J}=7.6 \mathrm{~Hz}, 1 \mathrm{H}), 8.13(\mathrm{~s}, 1 \mathrm{H}), 7.69-7.72(\mathrm{~m}$, $2 \mathrm{H}), 7.50(\mathrm{t}, \mathrm{J}=7.3 \mathrm{~Hz}, 1 \mathrm{H}), 7.38(\mathrm{~d}, \mathrm{~J}=7.6 \mathrm{~Hz}, 2 \mathrm{H})$, $7.25(\mathrm{~d}, \mathrm{~J}=8.6 \mathrm{~Hz}, 2 \mathrm{H}) \mathrm{ppm}$; Anal.Calcd.for $\mathrm{C}_{14} \mathrm{H}_{8} \mathrm{Cl}_{2} \mathrm{~N}_{2} \mathrm{O}$ : C,57.77; $\mathrm{H}, 2.79 ; \mathrm{Cl}, 24.36 ; \mathrm{N}, 9.62$. Found:C, 57.92; H,3.02; N, 9.35; MS(EI): $\mathrm{m} / \mathrm{z}(\%)$, $291\left(\mathrm{M}^{+}\right)$.

\section{3-(pyridin-2-yl)-4(3H)-quinazolinone (7i)} $\mathrm{mp}\left({ }^{\circ} \mathrm{C}\right)^{18} 132-133 \mathrm{IR}(\mathrm{KBr}): 3061,1684$, 1608,1474, 1435, 1328, 1290, 1256, 915, 870, 771 $\mathrm{cm}^{-1}{ }^{1} \mathrm{HNMR}$ ((300MHz, DMSO-d $)$; $8.65(\mathrm{t}, \mathrm{J}=7.3 \mathrm{~Hz}$, $2 \mathrm{H}), 8.40(\mathrm{~s}, 1 \mathrm{H}), 7.90-7.92(\mathrm{~m}, 2 \mathrm{H}), 7.78-7.84(\mathrm{~m}$, $2 \mathrm{H})$, 7.54-7.57 (m, $1 \mathrm{H}), 7.39-7.41(\mathrm{~m}, 1 \mathrm{H}) \mathrm{ppm}$; Anal.Calcd for $\mathrm{C}_{13} \mathrm{H}_{9} \mathrm{~N}_{3} \mathrm{O}: \mathrm{C}, 69.97 ; \mathrm{H}, 4.08 ; \mathrm{N}, 18.88$. Found: C, 69.88; H, 3.99; N, 18.95; MS(EI): m/z(\%), $223\left(\mathrm{M}^{+}\right)$.

\section{RESULTS AND DISCUSSION}

In an effort to develop more widely applicable methodology, in order to optimize the reaction conditions initially we have studied the efficacy of cobalt(II) chloride by taking catalytic amount of $10 \mathrm{~mol} \%$ and anthranilamide $(1 \mathrm{mmol})$ and benzaldehyde $(1.3 \mathrm{mmol})$ in acetonitrile $(5 \mathrm{~mL})$ as model reaction(3a) refluxed at $70{ }^{\circ} \mathrm{C}$ for $5 \mathrm{~h}$, the reaction gave corresponding 2-Phenyl-4(3H)quinazolinone $93 \%$ yield. (Table 3 , entry 1 ). In the absence of cobalt(II) chloride even up to $15 \mathrm{~h}$ no reaction was observed. The model reaction (3a) was performed in various solvents using cobalt(II) chloride as catalyst to identify the best medium for the reaction. A range of solvents such as $\mathrm{CHCl}_{3}$, 
DMF, THF, DCM, $\mathrm{H}_{2} \mathrm{O}$, DMSO, $\mathrm{CCl}_{4}$, and $\mathrm{CH}_{3} \mathrm{CN}$ were examined and acetonitrile emerged as the solvent of choice in terms of reaction kinetics and product yield (Table 1, entry 7 ). While comparing the effect of various catalysts on the model reaction (3a) we found that cobalt(II) chloride was more effective than other catalysts tested in terms of isolated yields (Table-2, (3a) entry 7). An optimum amount of $10 \mathrm{~mol} \%$ of cobalt(II) chloride is sufficient to carry forward the reaction.

Table 1: Screening of solvents for the synthesis of 2-phenyl$4(3 \mathrm{H})$ quinazolinone $(3 \mathrm{a})$ from anthranilamide $(1 \mathrm{mmol})$ aldehyde (1.3mmol) and 3-phenyl-4(3H)quinazolinones (4a) from anthranilic acid $(1 \mathrm{mmol})$ aniline $(1.3 \mathrm{mmol})$ and triethyl-orthoformate $(1.5 \mathrm{mmol})$ heating at $70^{\circ} \mathrm{C}$ in the presence of cobalt (II) chloride (10mol\%).

\begin{tabular}{lcccccc}
\hline \multirow{2}{*}{ Entry } & \multirow{2}{*}{ Solvent (5ml) } & \multicolumn{2}{c}{ Time (hours) } & & \multicolumn{2}{c}{ Yield (\%) } \\
\cline { 3 - 3 } \cline { 6 - 7 } & & $\mathbf{3 a}$ & $\mathbf{4 a}$ & & $\mathbf{3 a}$ & $\mathbf{4 a}$ \\
\hline \multirow{2}{*}{1} & $\mathrm{CHCl}_{3}$ & 9 & 8 & & 40 & 45 \\
2 & $\mathrm{DMF}$ & 9 & 8 & & 50 & 56 \\
3 & $\mathrm{THF}$ & 9 & 8 & & 45 & 55 \\
4 & $\mathrm{CCl}_{4}$ & 10 & 9 & & 55 & 50 \\
5 & $\mathrm{H}_{2} \mathrm{O}$ & 12 & 12 & & 50 & 48 \\
6 & $\mathrm{DMSO}$ & 9 & 9 & & 50 & 50 \\
7 & $\mathrm{CH}_{3} \mathrm{CN}$ & 5 & 6 & & 93 & 95 \\
8 & $\mathrm{No} \mathrm{solvent}$ & 12 & 12 & & $\mathrm{NR}^{*}$ & $\mathrm{NR}^{*}$ \\
\hline
\end{tabular}

${ }^{*}$ NR: No Reaction

Table 2: Screening of various catalysts (10mol\%)as standard for the synthesis of 2-phenyl-4(3H) quinazolinone (3a) from anthranilamide $(1 \mathrm{mmol})$ aldehyde $(1.3 \mathrm{mmol})$, acetonitrile $(5 \mathrm{~mL})$ and 3-phenyl-4(3H)quinazolinones (4a) from anthranilic acid $(1 \mathrm{mmol})$, triethyl orthoformate $(1.5 \mathrm{mmol})$ aniline $(1.5 \mathrm{mmol})$, acetonitrile $(5 \mathrm{~mL})$ and heating at $70^{\circ} \mathrm{C}$

\begin{tabular}{llcccc}
\hline \multirow{2}{*}{ Entry } & Catalyst (10mol\%) & \multicolumn{2}{c}{ Time (hours) } & \multicolumn{2}{c}{ Yield (\%) } \\
& & 3a & 3a & 4a & 4a \\
\hline 1 & $\mathrm{Al}\left(\mathrm{NO}_{3}\right)_{3} \cdot 3 \mathrm{H}_{2} \mathrm{O}$ & 9 & 9 & 40 & 45 \\
2 & $\mathrm{Ni}\left(\mathrm{NO}_{3}\right)_{3} \cdot 3 \mathrm{H}_{2} \mathrm{O}$ & 9 & 9 & 45 & 50 \\
3 & $\mathrm{Nd}\left(\mathrm{NO}_{3}\right)_{3} \cdot 3 \mathrm{H}_{2} \mathrm{O}$ & 8 & 8 & 60 & 65 \\
4 & $\mathrm{Zn}\left(\mathrm{NO}_{3}\right)_{2} \cdot 3 \mathrm{H}_{2} \mathrm{O}$ & 8 & 8 & 65 & 70 \\
5 & $\mathrm{Cu}-\mathrm{Proline}$ & 8 & 9 & 35 & 45 \\
6 & $\mathrm{Zn}-\mathrm{Proline}$ & 9 & 9 & 40 & 46 \\
7 & $\mathrm{CoCl}$ & 5 & 6 & 93 & 95 \\
8 & $\mathrm{TBABr}$ & 9 & 8 & 25 & $\mathrm{NR}$ \\
9 & $\mathrm{~K} \mathrm{~K}_{4} \mathrm{Fe}_{2}(\mathrm{CN})_{6}$ & 9 & 9 & 35 & 35 \\
10 & $\mathrm{Rh}\left(\mathrm{NO}_{3}\right)_{3} \cdot 3 \mathrm{H}_{2} \mathrm{O}$ & 9 & 8 & 45 & 60 \\
11 & $\mathrm{Ce}\left(\mathrm{NH}_{4}\right)_{2} \cdot 3 \mathrm{H}_{2} \mathrm{O}$ & 9 & 9 & 60 & 55 \\
12 & $\mathrm{Na} \mathrm{MoO}_{4} \cdot 2 \mathrm{H}_{2} \mathrm{O}$ & 9 & 9 & $\mathrm{NR}$ & 25 \\
\hline
\end{tabular}

* NR: No Reaction 
Encouraged by the results obtained for benzaldehyde (3a) we generalized the reaction scope for a number of other structurally divergent aromatic aldehydes, aliphatic aldehydes such as (propanaldehyde, butyraldehyde, and acetaldehyde, Table 3 , entry $1 \mathrm{j}, 1 \mathrm{k}, 1 \mathrm{l}$ ) heterocyclic aldehydes (furan-2 aldehyde, thiophene-2 aldehyde Table 3, entry 1f, $1 \mathrm{~m}$ ). In general electron rich counter parts such as hydroxy, methoxy, methyl groups require less reaction time than those of electron withdrawing groups such as (nitro group, halide group) were employed and reacted well to give the corresponding 2-substituted $4(3 \mathrm{H})$ quinazolinones.

Encouraged by the results obtained for benzaldehyde ( $3 a$ ) we generalized the reaction scope for a number of other structurally divergent aromatic aldehydes, aliphatic aldehydes such as (propanaldehyde, butyraldehyde, and acetaldehyde, Table 3 , entry $1 \mathrm{j}, 1 \mathrm{k}, 1 \mathrm{l}$ ) heterocyclic aldehydes (furan-2 aldehyde, thiophene-2 aldehyde Table 3, entry 1f, $1 \mathrm{~m}$ ). In general electron rich counter parts such as hydroxy, methoxy, methyl

Table 3: Synthesis of 2-substituted $-4(3 \mathrm{H})$ quinazolinones by the reaction of aliphatic, aromatic and hetero aromatic aldehydes with anthranilamide, catalyzed by cobalt (II) chloride in acetonitrile at $70^{\circ} \mathrm{C}$

\begin{tabular}{|c|c|c|c|c|c|c|}
\hline Entry & Aldehyde (2) & uct $(3 a-3 m)^{a}$ & Time (hours) ${ }^{\mathrm{C}}$ & Yield (\%) & \multicolumn{2}{|c|}{$\mathrm{mp}\left({ }^{\circ} \mathrm{C}\right) /$ Reference } \\
\hline $1 \mathrm{a}$ & $\mathrm{C}_{6} \mathrm{H}_{5} \mathrm{CHO}$ & $3 a$ & 5 & 93 & $237-239$ & 25 \\
\hline $1 \mathrm{~b}$ & $4-\mathrm{OCH}_{3} \mathrm{C}_{6} \mathrm{H}_{4} \mathrm{CHO}$ & $3 b$ & 5 & 90 & $243-244$ & 25 \\
\hline $1 \mathrm{c}$ & $4-\mathrm{OH}-3-\mathrm{OCH}_{3} \mathrm{C}_{6} \mathrm{H}_{3} \mathrm{CHO}$ & $3 c$ & 5 & 84 & $267-268$ & 25 \\
\hline $1 d$ & $4-\mathrm{OHC}_{6} \mathrm{H}_{4} \mathrm{CHO}$ & $3 d$ & 5 & 80 & $>300$ & 25 \\
\hline $1 e$ & $4-\mathrm{N}\left(\mathrm{CH}_{3}\right)_{2} \mathrm{C}_{6} \mathrm{H}_{4} \mathrm{CHO}$ & $3 e$ & 6 & 83 & $240-241$ & 25 \\
\hline If & $\mathrm{C}_{4} \mathrm{H}_{3} \mathrm{OCHO}$ & $3 f$ & 7 & 78 & $219-222$ & 25 \\
\hline $1 \mathrm{~g}$ & $4-\mathrm{ClC}_{6} \mathrm{H}_{4} \mathrm{CHO}$ & $3 g$ & 7.5 & 75 & $>300$ & 25 \\
\hline $1 \mathrm{~h}$ & $4-\mathrm{BrC}_{6} \mathrm{H}_{4} \mathrm{CHO}$ & $3 \mathrm{~h}$ & 6 & 80 & 293-295 & 25 \\
\hline $1 \mathrm{i}$ & $4-\mathrm{NO}_{2} \mathrm{C}_{6} \mathrm{H}_{4} \mathrm{CHO}$ & $3 \mathbf{i}$ & 7 & 78 & $>300$ & 25 \\
\hline $1 \mathrm{j}$ & $\mathrm{CH}_{3} \mathrm{CH}_{2} \mathrm{CHO}$ & $3 \mathbf{j}$ & 7 & 84 & $230-232$ & 25 \\
\hline $1 \mathrm{k}$ & $\mathrm{CH}_{3} \mathrm{CH}_{2} \mathrm{CH}_{2} \mathrm{CHO}$ & $3 K$ & 7.5 & 83 & $200-203$ & 25 \\
\hline 11 & $\mathrm{CH}_{3} \mathrm{CHO}$ & $3 \mid$ & 7 & 82 & $240-242$ & 25 \\
\hline $1 \mathrm{~m}$ & $\mathrm{C}_{4} \mathrm{H}_{3} \mathrm{SCHO}$ & $3 m$ & 7.5 & 80 & $219-221$ & 20 \\
\hline
\end{tabular}


groups require less reaction time than those of electron withdrawing groups such as (nitro group, halide group) were employed and reacted well to give the corresponding 2-substituted $4(3 \mathrm{H})$ quinazolinones.

In view of the above results obtained for the synthesis of 2-substituted-4(3H) quinazolinones, we wish to explore the use of cobalt (II) chloride as catalyst for the synthesis of 3substituted-4(3H)quinazolinone (scheme-2). The treatment of anthranilic acid, triethyl orthoformate $\left(\mathrm{CH}(\mathrm{OEt})_{3}\right)$, primary amines, in acetonitrile as solvent and cobalt(II) chloride (10mol\%) as catalyst, heating at $70{ }^{\circ} \mathrm{C}$ for the time specified for each substrate in (Table-4) resulting in the formation of 3 substituted $4(3 \mathrm{H})$ quinazolinones.

In a similar fashion we studied the efficacy of different catalyst chosen (10mol\%) as standard on the model reaction (4a) by taking anthranilic acid, triethyl orthoformate, aniline, acetonitrile, $(5 \mathrm{~mL})$,

Table 4: Synthesis of 3-substituted 4(3H)quinazolinones by the reaction of anthranilic acid (4), $\mathrm{HC}(\mathrm{OEt})_{3}(5)$, aromatic amines, catalyzed by cobalt (II) chloride in acetonitrile at $70^{\circ} \mathrm{C}$.

\begin{tabular}{|c|c|c|c|c|c|c|}
\hline Entr & $\mathrm{R}-\mathrm{NH}_{2}(\mathbf{6})$ & Produc(7a-7o $)^{a}$ & Time (hours) ${ }^{b}$ & Yield $(\%)^{c}$ & \multicolumn{2}{|c|}{$\mathrm{mp}\left({ }^{\circ} \mathrm{C}\right) /$ Reference } \\
\hline $2 a$ & $\mathrm{C}_{6} \mathrm{H}_{5} \mathrm{NH}_{2}$ & $7 a$ & 6 & 95 & 139 & 19 \\
\hline $2 b$ & $2-\mathrm{ClC}_{6} \mathrm{H}_{4} \mathrm{NH}_{2}$ & $7 b$ & 7 & 86 & 178 & $14 b$ \\
\hline $2 c$ & 4- $\mathrm{BrC}_{6} \mathrm{H}_{4} \mathrm{NH}_{2}$ & $7 c$ & 7 & 85 & 186 & 19 \\
\hline $2 d$ & 2,4- $\left(\mathrm{CH}_{3}\right)_{2} \mathrm{C}_{6} \mathrm{H}_{3} \mathrm{NH}_{2}$ & $7 d$ & 7 & 82 & Liquid & $14 b$ \\
\hline $2 e$ & $4-\mathrm{OCH}_{3} \mathrm{C}_{6} \mathrm{H}_{4} \mathrm{NH}_{2}$ & $7 e$ & 6 & 80 & 195 & 19 \\
\hline $2 f$ & 4- $\mathrm{CH}_{3} \mathrm{C}_{6} \mathrm{H}_{4} \mathrm{NH}_{2}$ & $7 f$ & 6 & 85 & 147 & 19 \\
\hline $2 g$ & 4-ClC ${ }_{6} \mathrm{H}_{4} \mathrm{NH}_{2}$ & $7 \mathrm{~g}$ & 7 & 82 & 192 & $14 \mathrm{~b}$ \\
\hline $2 \mathrm{~h}$ & $2,5-\mathrm{Cl}_{2} \mathrm{C}_{6} \mathrm{H}_{3} \mathrm{NH}_{2}$ & $7 \mathrm{~h}$ & 7 & 80 & 147 & 19 \\
\hline $2 i$ & $2,6-\mathrm{Cl}_{2} \mathrm{C}_{6} \mathrm{H}_{3} \mathrm{NH}_{2}$ & $7 i$ & 7.5 & 78 & 253 & 19 \\
\hline $2 \mathrm{j}$ & $3-\mathrm{NO}_{2} \mathrm{C}_{6} \mathrm{H}_{4} \mathrm{NH}_{2}$ & $7 \mathbf{j}$ & 7 & 78 & $200-202$ & $14 b$ \\
\hline $2 \mathrm{k}$ & $2-\mathrm{NH}_{2} \mathrm{C}_{5} \mathrm{H}_{4} \mathrm{~N}$ & $7 k$ & 7 & 80 & $131-132$ & 18 \\
\hline 21 & 3- $\mathrm{OCH}_{3} \mathrm{C}_{6} \mathrm{H}_{4} \mathrm{NH}_{2}$ & 71 & 7 & 80 & 196 & $14 b$ \\
\hline $2 m$ & $4-\mathrm{NO}_{2} \mathrm{C}_{6} \mathrm{H}_{4} \mathrm{NH}_{2}$ & $7 m$ & 7 & 80 & $199-200$ & $14 b$ \\
\hline $2 n$ & $3,4-\mathrm{Cl}_{2} \mathrm{C}_{6} \mathrm{H}_{3} \mathrm{NH}_{2}$ & $7 n$ & 7.5 & 75 & $164-168$ & 19 \\
\hline 20 & $2,4-\mathrm{Cl}_{2} \mathrm{C}_{6} \mathrm{H}_{3} \mathrm{NH}_{2}$ & 70 & 8 & 78 & $232-234$ & 19 \\
\hline
\end{tabular}

\footnotetext{
${ }^{\mathrm{a}}$ All the products are characterized by spectral analysis ${ }^{\mathrm{b}}$ refluxed at $70^{\circ} \mathrm{C} \quad{ }^{\mathrm{c}}$ isolated yields
} 
<smiles></smiles>

Scheme 1: Synthesis of 2-substituted-4(3H)quinazolinones<smiles>[R]c1cccc(-n2cnc3ccc(CC(C)C)cc3c2=O)c1[R]</smiles>

Scheme 2: Synthesis of 3-substituted-4(3H)quinazolinones

heating at $70^{\circ} \mathrm{C}$ for 6 hours results in the formation of 3-phenyl-4(3H)quinazolinone in $95 \%$ yield (Table-2, (4a) entry 7). The results shown that cobalt(II) chloride is emerged as best catalyst both in terms of reaction time and yields. Model reaction (4a) is screened for the best solvent by taking various solvents and acetonitrile is emerged as the best solvent (Table-1 $4 \mathrm{a}$, entry 7 ). Various 3 substituted $4(3 \mathrm{H})$ quinazolinone were prepared by using structurally varied anilines including pyridine2-amine (Table-4 entry $2 \mathrm{k}$ ). All the 2-substituted$4(3 \mathrm{H})$ quinazolinones and 3 -substituted$4(3 \mathrm{H})$ quinazolinones are well characterized by spectral analysis and with authentic samples.

\section{CONCLUSION}

In conclusion we have developed clean and efficient alternative protocols for the synthesis of 2-substituted-4(3H)quinazolinones from anthranilamide, aldehydes and synthesis of 3substituted- $4(3 \mathrm{H})$ quinazolinones from three component reaction of anthranilic acid, triethyl orthoformate, primary amines. The notable feature of these methodologies are by using a mild, inexpensive, easily available, cobalt (II) chloride as catalyst. We believe that this methodology will be a valuable addition for the synthesis of 2substituted-4 $(3 \mathrm{H})$ quinazolinones and 3 substituted-4 $(3 \mathrm{H})$ quinazolinones which are important synthetic interest because of their pharmacological and therapeutic properties such as anti-inflammatory, antiviral, anticancer activities etc.....

\section{ACKNOWLEDGEMENTS}

Dr. Aayesha Nasreen thanks Jazan University, for giving her an opportunity to continue research.

\section{REFERENCES}

1. Ghorab, M. M.; Farmco, 2000; 55: 249.

2. Bradly, D. S.; Tetrahedron Lett., 2001; 42: 1851.

3. Kumar, A.; Tyagi, M.; Shrivasthava,V.K.; Indian. J. Chem., 2003; 42B: 2142.

4. Brollosy-El.; Megeed- Abdel, N. R.; Genady, M. F.; Alexandria A. R. ; J. Pharm. Sci 2003; 17(1): 17.
5. Shab, B. R.; Bhatt, J. J.; Patel, H. H.; Undavia, N. K.; Trivedi, P. B.; Desai, N.C. Indian. J. Chem., 1995; 34B: 201.

6. Khili, M. A.; Soliman, R.; Furghuli, A. M.; Bekhit, A. A. Arch. Pharm, 1994; 27: 327.

7. Shivaram, H. B.; Padmaja, M. T.; Shivnanda, M. K.; Akbarali, P. M. Indian. J. Chem., 1998; 37B: 715. 
8. Hess, H. J.; Cronin, T. H.; Scriabine, A. J. Med. Chem., 1968; 11: 140.

9. Aziza, M. A.; Nassar, M. W.; Abdel Hamide, S. G.; El-Hakim, A. E.; EL-Azab, A. S. Indian. J. Heterocycl. Chem. 1996; 6(1): 25.

10. a) Pandey, V. K.; Pathak, L. P.; Mishra, S. K. Indian. J. Chem., 2005; 44B: 1940.

b) Pattanaik, J. M.; Paranaik, M.; Bhatta, D. Indian. J. Chem., 1998; 37B: 1304.

11. Acharyulu, P.V.R.; Dubey, P.K.; Prasada Reddy, P.V.V.; Suresh,T. ARKIVOC (xi) 2008; 104 .

12. Rewcastle, GW.; Denny W. A.; Bridges A. J.; Zhou, H.; Cod, R.; McMichael,A.; Fry, D.W, J. Med.Chem., 1995; 38: 3482.

13. Rosowsky, A.; Mota, C. E.; Wright, J. E.; Queener, S. F. J.Med.Chem.,1994; 37: 4522.

14. a) Jiang, J. B.; Hesson, D. P.; Dusak, B. A.; Dexter, D. L.; Kang, G. L.; Hamel, E. J. Med. Chem., 1990; 33: 1721.

b) Gnana ruba priya, M. International Journal of Pharma \& Bio Sciences, 2011; 2(1): 295.

15 a) Onaka, T. Tetrahedron Lett. 1971; 4387.

b) T. Kametani, T. Loc, C.V.; Higa,T.; Koizumi, M.; Ihara, M.; K. Fukumoto, K.; J. Am. Chem. Soc. 1977; 99, 2306.

c) Mori, M. H.; Kobayashi, H.; Kimura, M.; Ban,Y. Heterocycles, 1977; 23: 2803.

d) Sauter, F.; Frohlic, J.; Blasl, K.; Gewald, K. Heterocycles, 1985; 40: 851.

e) Majo, V.J.; Perumal, P.T.; Tetrhedron Lett. 1996; 37: 5015.

f) Prasad, M.; Chen, L.; Repic, O.; Blacklock, T.J.; Synth. Commun. 1998; 28: 2125.

g) Connolly, D.J.; Guiry, P.J. Synlett, 2001; 1707.

h) Wang, L. Xia, J. F. Qin, F.; Qian, C.; Sun, J. Synthesis, 2003; 1241.

i) Das, B.; Banerjee, J.; Chem.Lett. 2004; 33: 960.

16. a) S. E. Lopez, M. E. Rosales, N. Urdaneta, M. V. Godoy,J. E. Charris, J. Chem. Res., Synop., 2000; 6: 258.

b) J. J. Naleway, C. M. J. Fox, D. Robinhold, E. Terpetsching, N. A. Olsen, R. P. Haugland, Tetrahedron Lett. 1994; 35: 8569.

c) K. S. Deepthi, D. S. Reddy, P. P.Reddy, P. S. N. Reddy, Indian J. Chem., Sect. B 2000; 39: 220.

17.

a) Adharvana C. M.; Shobha, D.; Mukkanti,
K.; Catalysis Communications, 2006; 7: 787.

b) Lingaiah,V. B.; Ezikiel, G. T.Yakaiah; Reddy, V. G.; Rao, P. S. Synlett, 2006; 2507.

18. Bi Jing, Xiao.; Li, Z.; Pan, X.; Shi, Y.C.; Journal of the Chinese Chemical Society, 2008; 55: 1145.

19. Ighilahriz Karima,; Boutemeur, B.; Chami, F.; Rabia, Cherifa.; Hamdi, M.; Hamdi S. M. Molecules, 2008; 13: 779.

20. Raid, J.; Abdel-Jalil.; Voelterband, W.; Saeed, M.; Tetrahedron Letters, 2004; 45: 3475.

21. Narasimhulu, M.; Mahesh, K.C.; Srikanth Reddy, T.; Rajesh, K.; Venkateswarlu,Y.; Tetrahedron Letters, 2006; 47: 4381.

22. Khosropour, A. R.; Baltorka, I. M.; Ghorbankhani, Hamid. ; Tetrahedron Letters, 2006; 47: 3561.

23. Li, F.; Feng, Y.; Meng, Q.; Li, W.; Li, Z.; Wang, Q.; Tao, F.; ARKIVOC, (i ), 2007; 40.

24. Adib, M.; Sheikhi,E.; Bijanzadeh, H. R. Synlett, 2012; 85.

25. a) Wang G-W.; Miao,C. B.; Kang, H. Bulletin of the chemical society of japan, 79(9), 1426.

b) Durgareddy G. K.; Ravikumar, R.; Ravi, S.; Adapa S. R. J. Chem. Sci. 2013; 125: 175.

c) Errede, L. A.; Martinucci, P. D.; McBrady, J. J. J. Org.Chem., 1980; 45: 3009.

d) Zentmyer, D. T.; Wagner, E. C.; J. Org.Chem., 1949; 14: 967.

26. Rad-Moghadam, K.; Khajavi, S. M. J. Chem. Res., (S) 2006; 702.

27. Nasreen, A. Tetrahedron Lett. 2013; 54: 3797.

28. Nasreen, A. Asian journal of chemistry, 2013; 25: 7535 .

29. a) Varala, R.; Nasreen, A.; Ramu, E.; Adapa, S. R. Tetrahedron Lett. 2007; 48: 69.

b) Varala, R.; Nasreen, A.; Adapa, S. R. Can. J. Chem., 2007; 85: 1.

c) Nasreen, A.; Varala, R.; Adapa, S. R. J. Heterocycl. Chem. 2007; 44: 1.

30. a) De, S.K.; Tetrahedron Lett. 2004; 45: 1035.

b) De, S.K. Beilstein Journal of Organic Chemistry. ttp://bjoc.beilstein-journals.org/ content/1/1/8, 2005.

31. Iqbal, J.; Srivastava, R.R. Tetrahedron Lett. 1991; 32: 1663.

32. Velusamy, S.; Borpuzari, S.; Punniyamurthy, T. Tetrahedron, 2005; 61: 2001. 